\title{
LA FORMACIÓN DE LOS ESTUDIANTES EN HUMANIDADES Y CIENCIAS SOCIALES EN AMÉRICA LATINA
}

Notas de investigación

\begin{abstract}
¿Permaneced fieles a la tierra, hermanos, con el poder de vuestra virtud! „Vuestro amor generoso y vuestro conocimiento deben servir al sentido de la tierra! Os lo ruego encarecidamente ;No permitáis que alcen el vuelo, y abandonen las cosas terrenas, y con sus alas golpeen contra paredes eternas!

¡Ay, en todo tiempo ha habido mucha virtud que volando se extraviói i Conducid, como yo, la virtud extraviada de regreso a la tierra, de regreso al cuerpo y a la vida; para que dé a la tierra su sentido, un sentido de hombres para hombres!
\end{abstract}

Asi Habla Zaratustra F. Nietzsche

\section{Resumen}

Este artículo intenta delinear la explicación de una nueva actitud frente al conocimiento en lo que concierne a las humanidades $y$ a las ciencias sociales.

Tratamos de abordar el tema del conocimiento como experiencia sensible, lo cual no quiere decir que estamos proponiendo un distanciamiento de los estudios académicos o de las aulas universitarias, sino que ante las nuevas y complejas realidades se hace necesario repensar el proceso de formación de los graduados en estas áreas para abordar las nuevas situaciones y los campos profesionales a los que se deberán enfrentar.

Por el contrario, somos defensores del estudio universitario y de los centros académicos latinoamericanos. Como creemos que los alumnos "deben estudiar más", también estamos convencidos de que docentes y alumnos deben "desacartonarse" en el ámbito académico.

De este modo proponemos y desarrollamos algunas categorías de análisis enhebradas con experiencias de la cotidianidad en el campo profesional, y un anexo final que narra una experiencia de la propuesta que hacemos en este artículo: la universidad vivencial.

Palabras clave: Formación, campo profesional, ciencias sociales.

\footnotetext{
*Profesor de la Universidad Nacional de la Plata, Argentina. E-mail: cgtello@infovia.com.ar

Texto recibido el 21 de febrero de 2005 y aprobado el 28 de abril de 2005.
} 


\title{
THE FORMATION OF THE STUDENTS IN HUMANITIES AND SOCIAL SCIENCES IN LATIN AMERICA. Notes of Investigation
}

\begin{abstract}
This article intent as opposed to delineate the explanation of a new attitude the knowledge in which it concerns the humanities and social sciences.

We tried to approach the subject of the knowledge like sensible experience, which does not mean that we are proposing a spacing of the academics studies or the university classrooms. But that before the new and complex realities becomes necessary to return to think the process of formacion of the graduated ones about these areas to approach the new situations and professional fields which you would be deferent to face.

On the contrary we are defending of the university study and of the Latin American academicos centers, as we think that the students "must study more" too we are convinced that educational and students must "uncouple" themselves in the academic ambit.

This way we propose and we developed some categories of analysis threaded with experiences of the routine character in the professional field and an annexed end that narrates an experience of the proposal that we do in this I article: the existential university.
\end{abstract}

Key words: Formation, professional field, social sciences.

\section{Presentación}

Este artículo cuestiona el modo en que es abordado el conocimiento en el área de las ciencias humanas en las universidades argentinas y latinoamericanas, ya que consideramos, parafraseando a Nietzsche, que el modo en que se adquiere no responde a las necesidades de la tierra, y su vuelo golpea contra paredes eternas sin dar sentido a ella. De tal forma abordaremos un tema de carácter polémico: el conocimiento como experiencia sensible, así que asumiendo esta afirmación pasaríamos a un segundo plano: el dilema entre teoría y práctica no sería más que una falsa conjunción.

En estas notas de investigación no pretendemos tener demasiadas respuestas, dado que nuestro trabajo se encuentra en los inicios de la indagación. En tanto buscaremos algunas aproximaciones que podrán ser nuestras bases para seguir avanzando en el desarrollo de nuestro planteamiento.

Una de las grandes dificultades de la formación se observa en el momento de enfrentarse al ejercicio de su profesión, pero no podemos comenzar por alli, en este artículo sólo nos referiremos a nuestro primer avance: el proceso formativo.
Aquí no intentamos plantear un modelo epistemológico sino una actitud frente al conocimiento y a la forma en que se aprende a pensar la realidad en los centros universitarios. Uno de los grandes conflictos que observamos en el campo de las humanidades es la profunda influencia de las corrientes filosóficas positivistas y analíticas, los enfoques mecanicistas que amalgamaron y anquilosaron el desarrollo de múltiples perspectivas emergentes que luego se manifestaron ocultas bajo la raigambre desarrollista, que trajo consigo el tecnicismo pedagógico y la sociología científica, denominadas en la actualidad como neofuncionalismo liberal en las Humanidades, o neoliberalismo, entendiendo el desarrollismo de la década del sesenta como la "bisagra" del capitalismo actual.

\section{Introducción}

Destacados intelectuales han realizado investigaciones respecto a la relación teoría y práctica, y al respecto de la formación, pero en general no se abordó el modo como se adquiere el conocimiento y es allí, como hemos dicho, donde in tentaremos inmiscuirnos. A raíz de este planteamiento detectamos situaciones que podriamos sintetizar en las palabras de un dirigente piquetero ante un licenciado en Trabajo Social que había concurrido a una marcha para acompañarlos, a la cual se le increpó: "Siiii, Uds... Mucha teoría... mucha teoría, pero al momento de estar frente a los problemas o resolver cuestiones concretas... no bajan a la tierra". "No tienen los pies sobre la tierra"1.

\section{Desde dónde realizamos nuestro planteamiento}

Nuestro postulado está totalmente alejado de las posturas psicologistas; por tanto, intentaremos, como plantea Ricoeur ${ }^{2}$, "despsicologizar" la perspectiva, ya que la psicología educativa pareciera quedarse con la autoridad en este tipo de análisis, y es allí donde se manifiesta con mayor claridad la expresión hegemónica en las ciencias sociales actuales.

Hoy esta tendencia se manifiesta a través de la preponderancia que tienen las teorías cognitivas, Neurobiológicas y holográmicas en el campo del conocimiento. Nuestra pos-

${ }^{1}$ Respuesta dada de un dirigente del MTD a la licenciada en Trabajo Social, J.N.A.

${ }^{2}$ Ricoeur, P., "La acción entendida como un texto", en: Hermenéutica y acción. Docencia. Buenos Aires. 1988. 
tura surge de la teoría social crítica en el sentido habermasiano, pueto a que desde esta perspectiva, Habermas hace una crítica del positivismo y de la técnica científica.

Tomamos tres elementos centrales de la perspectiva habermasiana para posicionarnos en este tema:

- Crítica a la ciencia, mediante una oposición al empirismo y a la razón científico-técnica e instrumental, manifestada a través de una negación de la supuesta objetividad y neutralidad científicas. Denuncia, pues, la falsa objetividad científica.

- Crítica a la filosofía teórica tradicional por su abstracción y pura especulación, es decir, por hallarse al margen del cualquier contexto histórico-social y de las condiciones materiales que la sustentan.

- Filosofía del compromiso social, la transformación de la sociedad. Para ello se deberá partir de las condiciones materiales (socioeconómicas) e histórico-sociales que enmarcan tanto el pensamiento como la acción de los individuos.

Marcuse responde con la afirmativa, a nuestro interrogante:

"Los principios de la ciencia moderna estaban estructurados a priori de forma, que podian servir como instrumentos conceptuales para un universo de controles productivos que se ejercen automáticamente. El método científico que conducia cada vez a una dominación más eficiente de la naturaleza, proporcionó también tanto los conceptos puros como los instrumentos para una dominación cada vez más efectiva del hombre sobre el hombre a través de la dominación de la naturaleza...

Esta apelación a los imperativos técnicos sólo es posible porque la racionalidad de la ciencia y de la técnica ya es por su propia esencia una racionalidad del disponer, una racionalidad del dominio" J. Habermás, Ciencia y técnica como ideología,

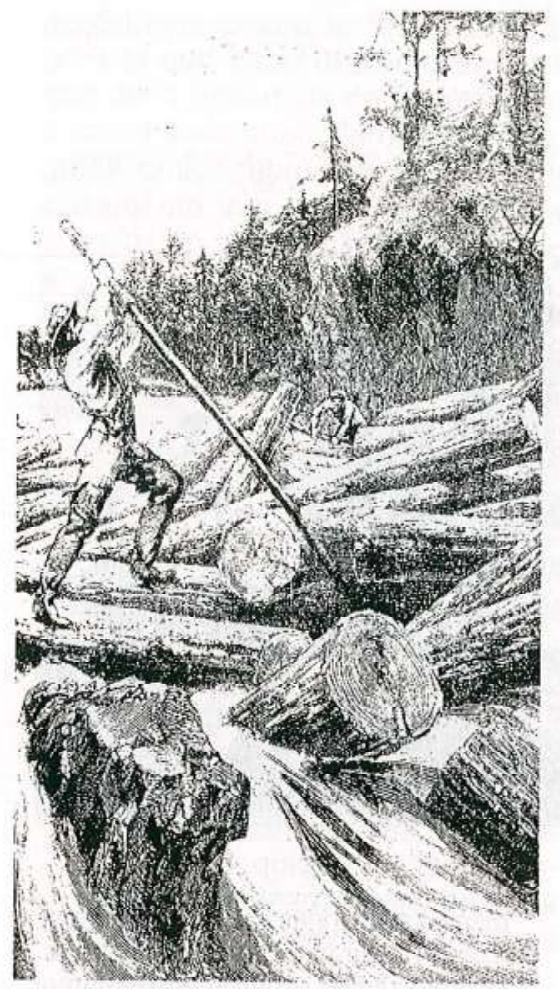

\section{Análisis}

El proceso de alfabetización no es solo cognoscitivo, también es sociale "ideológico". O sea, el proceso de alfabetización necesita atacar las causas de desigualdad en la sociedad.

Los niños que provienen de sectores oprimidos conozcan a través de esta pedagogía su propia realidad y liberación

P. Freire

Creo que la narración que mostramos a continuación muestra en qué sentido pensamos el conocimiento como intuición, acercándonos a la definición del conocimiento como experiencia sensible en los estudiantes universitarios de humanidades y ciencias sociales. Por tanto tomamos la anécdota del profesor Alexander Calandra, de la Universidad de Washington en Saint Louis:

\section{EL BARÓMETRO}

Hace algún tiempo me llamó un colega para preguntar si yo estaria dispuesto a servir como árbitro en la calificación de un examen. Parecía que él estaba dispuesto a ponerle cero al estudiante por su respuesta a una pregunta de física, mientras el alumno reclamaba que debía tener la máxima calificación, y que de hecho la tendría si el sistema no estuviese cargado en contra de los estudiantes. El profesor y el alumno se pusieron de acuerdo en someter la cuestión al juicio de un árbitro imparcial, y yo fui el escogido. Fui a la oficina del colega y leí la pregunta que decia asi: "Explique cómo determinar la altura de un edificio con la ayuda de un barómetro". La respuesta del estudiante fue: "'Lleve el barómetro hasta lo más alto del edificio, amárrele una cuerda larga, haga bajar el barómetro hasta la calle, súbalo de nuevo y mida el largo de la cuerda. El largo de la cuerda corresponde a la altura del edificio". Ahora, ésta es una respuesta muy interesante, pero ¿debería el estudiante obtener crédito por ella? "Yo señalé que el alumno de veras tenía un buen argumento para reclamar la máxima calificación, puesto que había respondido de forma correcta y completa. Sin embargo, si le hubiese dado el crédito total esto implicaria que el estudiante obtuviese muy alta calificación en el curso de física. Una alta calificación supuestamente certifica que el estudiante sabe bastante de física, pero la respuesta a la pregunta no confirma que asi fuera. Con este dilema en mente, sugeri que el estudiante tuviese una segunda oportunidad para responder la pregunta. No me sorprendió que mi colega estuviera de acuerdo, pero sí me sorprendió que el alumno aceptara de inmediato. Así pues le di seis minutos al estudiante para responder la pregunta, con la advertencia de que su respuesta debía mostrar algún conocimiento de la física. Al término de cuatro minutos no había escrito nada. Le pregunté si deseaba abandonar, dado que yo tenía otra clase que dar, pero él dijo que no, que no abandonaría, que tenía muchas posibles respuestas a la pregunta, y que solo estaba pensando cuál de ellas sería la más acertada. Me excusé por haberlo interrumpido y le pedí que siguiera adelante. En el minuto siguiente él rápidamente escribió su respuesta. Era ésta: "lleve el barómetro a lo más alto del edificio. Deje caer el barómetro. Midiendo el tiempo de caída con un cronómetro. Después, utilizando la fórmula $\mathrm{d}=1 / 2 \mathrm{at}^{2}$ (la distancia en caída equivale a la mitad de la aceleración por el cuadrado del tiempo trans- 


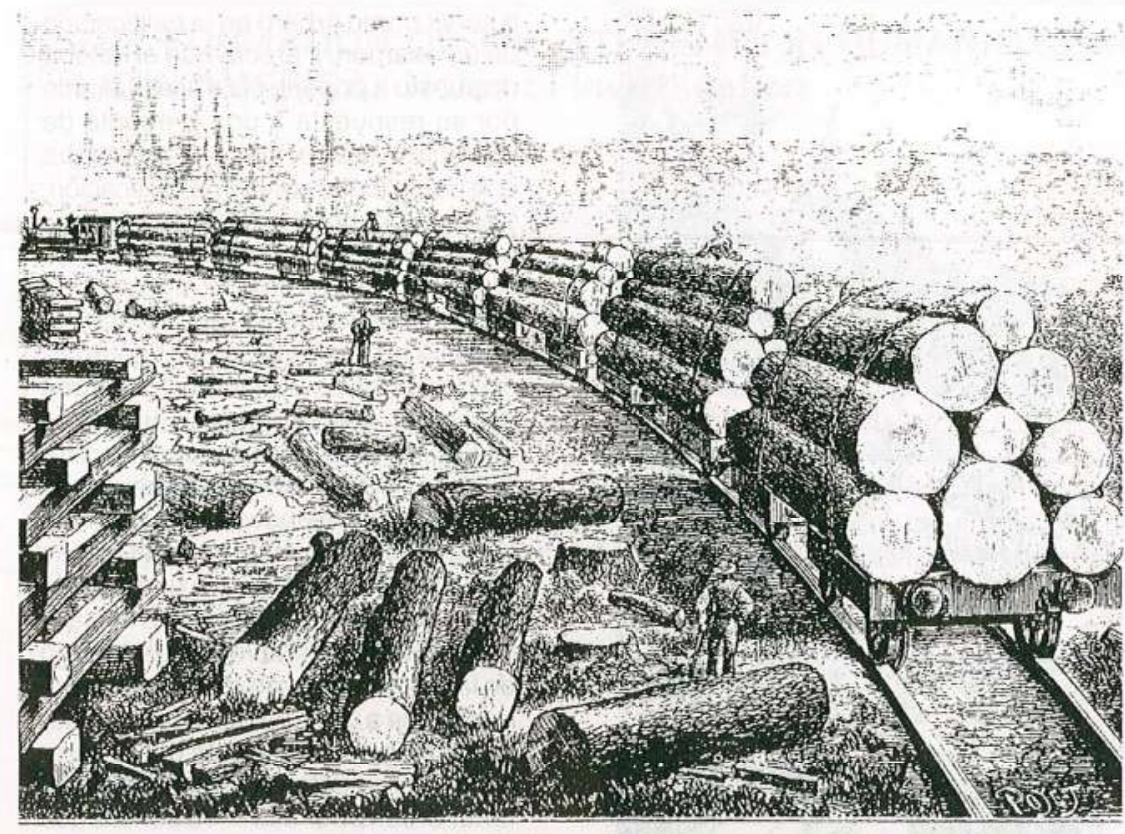

currido), calcule la altura del edificio". En este punto le pregunté a mi colega si él estaba dispuesto a declinar su calificación. Él hizo la concesión, y yo le di al estudiante casi la máxima calificación. Cuando salía de la oficina de mi colega, recordé que el estudiante había dicho que tenía varias respuestas al problema. "Ah sí, dijo el estudiante. Hay muchas formas de saber la altura de un edificio con la altura de un barómetro. Por ejemplo, uno podría sacar e barómetro en un día soleado y medir la altura del barómetro, el ancho de su sombra, y el largo de la sombra del edificio; y utilizando simple proporción, determinar la altura del edificio." "Bueno, dije, ¿y las otras? "Sí, dijo el estudiante. Hay una forma de medición básica que a usted le gustará. En este método, usted toma el barómetro y empieza a subir las escaleras. A medida que sube, coloca el barómetro sobre la base de cada escalón, marca con un lápiz la altura alcanzada, y vuelve a colocar e barómetro sobre la marca, hasta llegar al piso siguiente. La suma de los pisos le dará la altura del edificio en unidades barométricas. Es un método muy directo. Claro que si usted desea un método más sofisticado, puede amarrar el barómetro a una cuerda, hacerlo oscilar como un péndulo, y determinar el valor de g [la aceleración de la gravedad], a nivel de la calle y a nivel del punto más alto del edificio. Finalmente, concluyó: "si ustedes no me li- mitan a soluciones físicas al problema, existen muchas otras respuestas, tales como llevar el barómetro al primer piso y golpear en la puerta del conserje. Cuando él abra la puerta, usted le dice: Querido señor conserje, aquí tengo un fino barómetro, si usted me dice la altura del edificio, yo le doy este barómetro..."3.

Con esta narración queremos ilustrar cómo muchas veces a nuestros alumnos los Ilevamos al análisis mediante procedimientos y operaciones que deben cumplir la rigurosidad de la supuesta objetividad, dentro de determinados marcos y esquemas científicos o académicos que los legitiman

El problema lo plantean claramente Maturana y Varela "Si lo conocido atrae (y retiene en una 'fijación' de la verdad) justamente por ser terreno 'conocido' bajo el aval de poderosas y 'sagradas' tradiciones, al convertirlas en verdades absolutas ha-

\footnotetext{
${ }^{3}$ Adaptado de Current Science, Teacher's Edition, Vol. 49, № 14, Enero 6-10, 1994.

${ }^{4} \mathrm{H}$. Maturana y F. Varela, El árbol del conocimiento. Editorial Universitaria. Santiago de Chile. 1984, p. 15
}

cemos de tales certidumbres las mayores barreras en la comprensión social mutua", en clara coincidencia con E. Eisner (1987) cuando afirma 'El dogmatismo metodológico, incluso en nombre de la verdad puede poner trabas a la capacidad humana de conocer"5.

Ahora bien, éste no es un pronunciamiento feyerabendiano donde realizaremos un "tratado contra el método"6, y creemos que incurrir en este campo de la epistemología y sus diversos instrumentos de investigación nos detendrá en un camino que a los fines de este artículo no deseamos recorrer.

Una cuestión interesante cuando se discute acerca de estas asuntos del conocimiento en el ámbito universitario es que se realiza una escisión entre lo cognitivo y lo afectivo como estados distintos e independientes del sujeto que aprende, cuando en realidad ni siquiera podríamos afirmar que son interdependientes sino que aseguramos que son simultáneos porque son parte de la misma persona. Aquí se plantea uno de nuestros postulados: no partimos del objeto cognoscible sino de la persona que conoce, que es un sujeto integral bio-psíquico-socio-cultural-emocional.

Pero insistimos en entrenar a nuestros alumnos en la disociación "los entrenamos para que den la respuesta correcta: ¿Para qué sirve un barómetro?".

En nuestra línea de trabajo consideramos que no puede haber ninguna actividad cognitiva sin afectividad. Por eso lo que se logra actualmente en general no es conocimiento; conocer algo sin sensibilidad es no conocerlo. El que no "recorra el

${ }^{5}$ E. Eisner, Procesos Cognitivos y curricu 10. Capítulo II: El papel de los sentidos en la formación de los conceptos. Martinez Roca. Barcelona. 1987, p. 76.

${ }^{6}$ Véase P. K. Feyerband, Contra el método. Barcelona: Ariel, 1974 
cuerpo" no sería natural, por eso el conocimiento académico como se plantea en general es artificial.

Maturana y Varela ${ }^{7}$ afirman:

la dificultad del conocimiento humano está en reconocer lo que yo llamo el fenómeno de tautología cognoscitiva... Con los términos anteriores estoy designando el hecho que el universo de conocimiento, de experiencias, de percepciones del ser humano no es posible explicarlo desde una perspectiva independiente de ese mismo universo. El conocimiento humano sólo podemos conocerlo desde sí mismo.

Cuando pensamos en la sensibilidad que provoca el conocimiento, o viceversa, como estados simultáneos, lo hacemos desde los distintos sentimientos que se pueden despertar en un ser humano: aversión, adhesión, enojo, tristeza, alegría, etc. $Y$ en esta misma línea es interesante plantear que el conocimiento como experiencia sensible puede ser insípido o apagado, pero sensible al fin.

No existe teoría y práctica, conocimiento y experiencia. Existen los sentidos: allí están la vida, las emociones, los dolores, la risa, las lágrimas, las elucubraciones. No hay duda de que psicólogos de la educación tendrán múltiples respuestas a este postulado, pero nosotros decididamente tomamos otro camino. Nosotros afirmamos que el dilema entre teoría y práctica es un razonamiento falaz porque no existe tal distancia.

Toda esta cuestión depende de la concepción de conocimiento de la que se parte. Si lo hacemos de los modelos hegemónicos pospositivistas, arribaremos a esa brecha, pero si pensamos en una nueva perspectiva de las ciencias sociales donde concebimos al conocimiento como una experiencia sensible, no existirá tal cuestión. Nosotros hemos planteado nuestros

${ }^{7}$ Maturana y Valera, op. cit. postulados desde la teoría crítica, pero lo que cabe destacar aquí es que de la tendencia crítico-reflexiva o como se la suele llamar en pedagogía, crítico-reproductivista, surgen claramente tres líneas en cuanto a su relación teoría y práctica:

1. La propuesta por la conjunción investigación-acción, entre cuyos mayores exponentes podríamos situar a Elliot (1990).

2. La propuesta que considera la reflexión acerca de las prácticas sintetizando los aspectos cognitivos. Quien desarrolló esta cuestión con mayor despliegue en el ámbito pedagógico fue Escurra (1990).

3. La propuesta que considera la dimensión gnoseológica y reconoce la dimensión política de los actos educativos, aquí es indiscutible que quienes mayores esfuerzos realizaron por introducir esta reflexión son Carr y Kemis (1988).

Según venimos planteando, ninguna de las tres líneas coincide con nuestros postulados, ya que hay conjunción, reconocimiento, síntesis, pero no se toma a la teoría y a la práctica como un proceso integral indisociable, si no que es la conjunción de..., el reconocimiento de... o la síntesis de... Esta perspectiva plantea la relación entre teoría y práctica, que vendría a ser de mutua regulación, y la teoría no configura la práctica, sino que se construyen simultáneamente y se constituye en herramienta para la lectura y transformación de la realidad, como veremos con la universidad vivencial.

\section{El frío de las instituciones "congela" y "adormece" los sentidos}

En la actualidad el pensamiento académico, la solución de problemas, el desarrollo de tareas, deben ser actividades que han de seguir rigurosamente el método científico y una resolución técnica.
Al alumno se le exigirá, según los niveles alcanzados en sus estudios, mayor o menor rigurosidad en sus expresiones y tareas académicas. Hacia el final de las carrera serán todos menos rigurosos, permitirán producciones "creativas" porque supuestamente ya se ha "normalizado" al sujeto; está institucionalizado. La racionalidad técnica se basa en un conocimiento científico especializado que se debe "aprender" en los primeros años de la carrera, y esto permite "estar dentro" del campo académico.

Sólo se conoce en las universidades a través de la razón, de la cognición, sin el cuerpo. Así el conocimiento es artificial y el frío de las instituciones "congela" $y$ "adormece" los sentidos.

Lo anterior lleva a que en el momento en que los profesores universitarios realizamos un planteamiento o cualquier tipo de conjetura el alumno responda: "Pero Ud., profesor, ¿pregunta desde lo teórico o desde la práctica?", o lo que es más contradictorio para mi, cuando los alumnos plantean esta distancia entre la teoría y práctica como limitación académica, de la cual desean salir, pero están prisioneros de la cultura institucional que los ha ido moldeando durante años. La cultura es responsable de la corrosión del carácter, ya que existe una institucionalización cultural que moldea a docentes y alumnos, y de la cual debemos desprendernos y desnaturalizarnos.

Me avergüenzo cuando eścucho que la facultad debe abrir las ventanas para que "entre la realidad". Hay que abrir las ventanas porque tenemos personas cargadas de racionalidad técnica, intelectuales que no logran, por las propias representaciones sociales, dejarse invadir desde los sentidos. $Y$ que el texto los penetre desde alli, desde los nervios y que luego "suba a la cabeza". El conocimiento debe provocar sensibilidad, gusto, sabor, pero no lo aprendimos así. 


\section{Aproximaciones finales}

Nos encontramos ante la situación de que los modelos actuales de indagación de la realidad no nos son útiles para interpretarla, porque sencillamente en estos tiempos de cambio y transición los paradigmas poco a poco van quedando obsoletos y anacrónicos como modelos de análisis.

La realidad invade de tal manera que los instrumentos metodológicos y la forma de observar, en la cual nos esforzamos en seguir implementados por "otros" que poco tienen que ver con la realidad latinoamericana,

A los fines de ilustración tomamos la categoría de "encontrar la ranura" de Schon": "Los pitchers de la gran liga de béisbol hablan, por ejemplo, de la experiencia de "encontrar la ranura'. Adquieres una sensación de la pelota, un tipo de dominio que te permite repetir la misma cosa que hiciste antes. Cuando consigues una sensación de pelota..." se sigue con ella y emerge del conocimiento de los pitchers y de la reflexión de ese conocimiento sobre la acción. Es intuición, sensación, percepción, es conocimiento sensible. Pero cada "sensación de pelota" puede ser distinta ante "aparentes" situaciones similares de la realidad. Los pitchers saben muy bien la teoría: saben cómo flexionar las rodillas, como inclinar el cuerpo para que la pelota obtenga mayor impulso, pero lo más difícil es "encontrar la ranura": "La sensación de pelota".

Dejar que el conocimiento fluya en la sangre, "tener la sensación de pelota", la ilustración aquí citada no debe confundir nuestra definición; "la sensación de pelota" se puede encontrar en un libro. Se puede leer Pedagogía de la esperanza de Paulo

${ }^{8}$ D. Schön, El profesional reflexivo. Capítulo II: De la racionalidad técnica a la reflexión desde la acción. Barcelona: Paidós, p. 60.1998.
Freire, y se lo puede aprender desde el conocimiento "muerto" o desde el conocimiento sensible encontrando la "sensación de pelota" dejando que penetre en la piel, que fluya en la sangre, que sensibilice los sentidos.

La cientificidad está dada por criterios "no humanos", está dada por esa racionalidad instrumental-técnica que está por fuera del ser que ya hemos tratado de explicar o atribuir a determinadas corrientes psicológicas y pedagógicas.

Es imperioso construir ciencias humanas y sociales latinoamericanas, que no pierdan de vista la realidad social de su propia tierra, pero que no dejen de avanzar en sus estudios no hegemónicos. Creando la alternativa al país del Norte o dejando de mirar al Viejo Continente, de hecho nuestro artículo está cargado de referencias europeas y norteamericanas, ya que hemos tenido que acudir a ellas para construir nuestro postulado.

¿Qué clase de graduados estamos preparando? ¿Hacia dónde van estos profesionales con letra a la que le falta vida, con marcos de indagación que no responden a nuestra realidad latinoamericana?

La indeterminación debería ser el eje de formación docente, esa indeterminación que sólo es posible afrontar desde otra indeterminación: la propia. La de nuestro continente. Por eso no aceptamos epistemologías europeizantes constreñidoras de carácter, mientras los hijos de la tierra lloran.

\section{Para todo esto será necesario:}

1. Redefinir y analizar los conceptos de educación según cada circunstancia. Las categorías de formación y profesión variarán de acuerdo con las necesidades de la sociedad.

2. Apertura multidimensional de la realidad social y apertura de los sentidos para profundizar en su análisis, sin anticipar cuál será la función del graduado porque seguramente será distinta para la cual "se lo está preparando".

Soñamos con que el graduado en humanidades y ciencias sociales utilizará sus sentidos cargados de conocimientos para abordar dificultades, y tratará de resolverlas "embarrándose las manos" de realidad, y no dará respuestas "técnicas recetadas" barnizadas de profesionalidad adquirida durante su paso por la universidad.

Para reforzar la idea, parafraseamos un postulado de Apple (1986) cuando afirma que se ha abusado sobre el concepto de profesionalismo en la enseñanza y que esos postulados pueden conducir a un proceso de paulatina tecnificación de la enseñanza, y que la creciente proletarización del graduado puede ser identificada erróneamente como un símbolo del incremento del profesionalismo, ya que se puede identificar erróneamente profesionalismo con aspectos técnicos de intervención producidos externamente a la práctica.

\section{La experiencia de la universidad vivencial}

De algún modo es necesario ilustrar el planteamiento que estamos haciendo donde se conjuguen universidad, academia y conocimiento sensible. Existe una experiencia donde se intenta llevar a cabo lo que aquí planteamos y para eso desarrollará este apartado del artículo un amigo y excelente profesional que participa de dicha experiencia, el Licenciado Christian Beri ${ }^{9}$. Debemos

${ }^{9}$ Christian Beri es graduado de la Universidad Nacional de La Plata en la Licenciatura en Ciencias Naturales con orientación en Ecología. Tiene una especial preocupación por las cuestiones educativas articulando los saberes de su campo. Actualmente desempeña el cargo de consejero de la Unidad Pedagógica de la Facultad de Ciencias Naturales y Museo. Es profesor en 
tener en cuenta que esta experiencia se desarrolla fuera de las aulas universitarias, pero surge allí; no es una actividad de extensión, ellos quieren alejarse de esa categoría. Sin duda que la narración es importante pero para entender lo que se expresa en estos párrafos no hay mejor forma que vivir la experiencia.

La Universidad Vivencial (UV '04) es básicamente un curso-taller de intercambio técnico cultural para estudiantes y graduados universitarios latinoamericanos que se realizó en el año 2004 en el marco más amplio de la propuesta del Grupo Epsylon $^{10}$. Esta agrupación, conformada inicialmente por un grupo de estudiantes avanzados y profesionales, nace de la participación en la Universidad Abierta de Verano organizada en Santos (Brasil) en los meses de enero de 1999 y 2000. Motivados por este modo de intercambio con otros estudiantes y profesionales jóvenes trabajando con poblaciones locales, deciden organizar, planificar y coordinar una experiencia propia. La idea de base es la autoconstrucción de herramientas intelectuales y afectivas a través de una experiencia en Argentina para estudiantes de distintos puntos de Latinoamérica, con una particular modalidad de aprendizaje didáctico, ecológico y de acción comunitaria.

La UV se desarrolló del 4 al 30 de enero de 2004 en la ciudad de La Plata (Provincia de Buenos Aires) contando con la participación de 20 estudiantes universitarios de Argentina, Brasil, Colombia, Chile y $\mathrm{Pa}$ raguay. La mayoría pertenecia al campo de la arquitectura, un biólo-

varios institutos superiores de la Provincia de Buenos Aires en el área de la didáctica de las ciencias naturales, y ha realizado exposiciones en congresos respecto a "La articulación entre polimodal y Universidad". En la actualidad es miembro del equipo que está desarrollando la experiencia narrada. ${ }^{10}$ www.grupoepsylon.com.ar/info@grupo epsylon.com.ar go (quien transcribe) y una estudiante de relaciones exteriores.

Para llevar a cabo la experiencia, los integrantes de la UV trabajaron durante todo el mes con una comunidad de la ciudad de Berisso, específicamente de la tradicional Calle Nueva York, conocida en la región por su esplendor comercial y arquitectónico de antaño, pero actualmente convertida en una zona de conventillos en un franco deterioro, abandonada por la gestión municipal, y con una población de familias numerosas de inmigrantes, en su mayoría desocupados.

El esquema de intervención de la UV intenta situarse al margen de los modelos tradicionales: construir viviendas, realizar exposiciones, charlas, etc., ya que de este modo no se logra otra cosa que un gran workshop (trabajo práctico).

Se espera suplir la carencia de este tipo de proyectos en la propuesta curricular universitaria, contribuir a la formación de jóvenes profesionales capaces de actuar autogestivamente en sus propias comunidades y promover el mejoramiento de algún aspecto del entorno socioambiental de la comunidad, respondiendo a las necesidades del momento; por eso se debe trabajar sobre la percepción social.

Con el lema "La transformación de una sociedad en crisis", la propues- ta ofrece un espacio de enriquecimiento a nivel personal, tanto de las dimensiones del trabajo en equipo como aquellas respecto a la intervención, ya que el desarrollo de la percepción no anula la intervención, por el contrario, la intención es mejorarla.

Ambas se trabajan paralelamente, y esto implica la modalidad de convivencia efectiva del equipo de participantes que se alojan en una residencia, donde "viven" esta experiencia formativa. El alojamiento dista varios kilómetros del lugar de trabajo y prevé el viaje casi diario de los participantes. Es en el seno de la convivencia donde se genera el mutuo conocimiento y la integración, los debates y conferencias que aportan diferentes elementos para un marco teórico de referencia, las discusiones de los pasos por seguir, la autoevaluación, la discusión de las ideas y toda una serie de dinámicas dirigidas a cimentar las relaciones grupales manteniendo el foco en la comunidad donde se desarrollará el proyecto.

El intercambio y la intervención comunitaria se realizan a través de la experiencia directa en el lugar donde se encuentra la comunidad.

La metodología empleada para su desarrollo busca generar la percepción del equipo sobre las necesidades de los habitantes y su participación activa en un proyecto tendien-

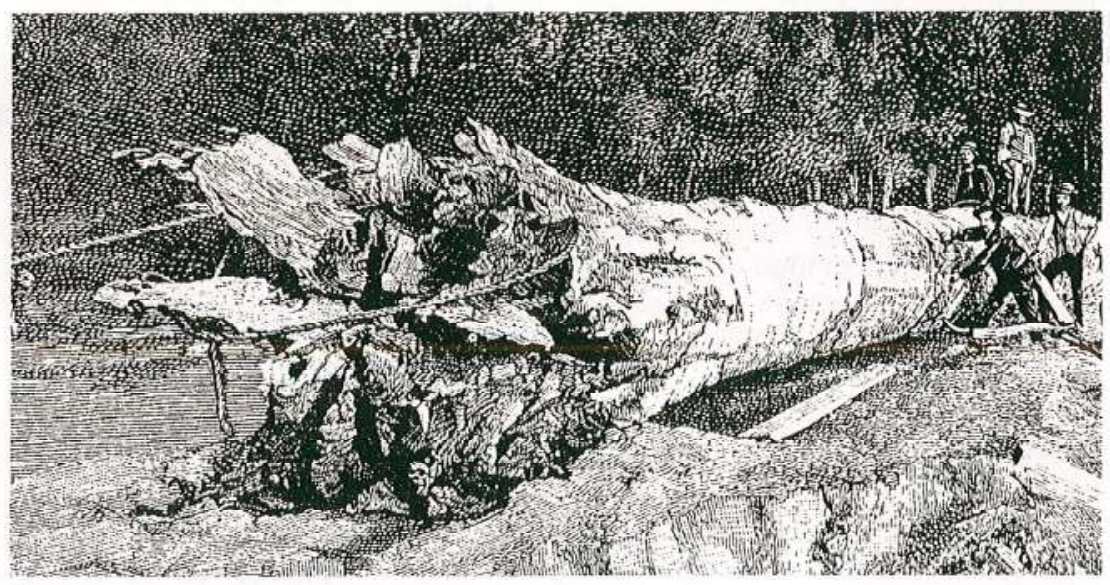


te a mejorar su calidad de vida, recuperando sus saberes y técnicas tradicionales, en este caso, de la comunidad de Berisso.

La metodología está basada en los siguientes pasos:

Percepción: percepción del individuo (superación de las propias dificultades), del trabajo colectivo inmediato (el intercambio de experiencias en el trabajo en equipo), del trabajo en comunidad (como se da el intercambio cultural en este contexto).

Información: la obtención de datos en los tres niveles de percepción: información sobre si mismo, sobre el otro (el trabajo en equipo) y sobre la comunidad. Métodos de abordaje, levantamiento de datos y clasificación.

Reflexión: a partir de la percepción y de la obtención de informaciones, construir la capacitación junto a los estudiantes para decidir el trabajo que se llevará a cabo.

Propuesta: la elaboración de un proyecto para la comunidad utilizando la gama de experiencias acumuladas en esta vivencia.

Acción: trabajar la dificultad de los estudiantes y jóvenes profesionales en viabilizar sus proyectos. A través de la construcción del proyecto elaborado en las etapas anteriores, el estudiante tendrá la posibilidad de aprender a construir junto con la comunidad y adaptar en la obra ciertas soluciones que no hayan sido desarrolladas correctamente en la fase proyectual. Lo que denominamos "el cambio sobre la marcha".

Las perspectivas futuras que se abren en función de este proyecto son muy vastas y se fundamentan en una experiencia que ha cumplido las expectativas de los organizadores. Estamos convencidos de que hay un trecho largo por recorrer y que la única manera de conocerlo es a través de "caminar" las sucesivas experiencias que cuentan tanto con una gran dosis de trabajo, creatividad e intuición, como de reflexión atenta al momento social que transitamos.

\section{BIBLIOGRAFÍA}

Авraham, A. (1986). El enseñante es también una persona. Barcelona. Gedisa.

Alliaud - Duschatzky (Comp.) (1992). Maestros, formación, práctica y transformación escolar Miño y Dávila.

Bernstein, R. J. (1982). La reestructuración de la teoría social y política. México: FCE.

Carr, W. y Kemis, S. (1988). Teoría Crítica de la enseñanza. La investigación acción en la formación del profesorado. Barcelona: Martinez Roca.

DaviNI, M. C. (1996). La formación docente en cuestión: política y pedagogía. Buenos Aires: Paidós.

Diez, J. A. y C.U. Moulines (1997). Fundamentos de filosofía de la ciencia. Barcelona: Ariel.
EISNer, E. (1987). Proceso Cognitivos y currículo. Barcelona: Martínez Roca.

LARROSA, J. (1990). El trabajo epistemológico en Pedagogía, Madrid: Labor.

Freire, P. (1992). La Educación como práctica de la libertad. Capital Federal: Siglo veintiuno editores.

GidDEns, A. (1987). Las nuevas reglas del método sociológico. Buenos Aires: Amorrortu.

Giroux, H. (1983). Teoría y resistencia en educación. Bergin and Garvey

Habermas, J. ( 1989) Teoria de la acción comunicativa. Buenos Aires: Taurus.

Habermas, J. (1985). Conocimiento e Interés. Buenos Aires: Taurus.

Hempel, C. G. (1973). Filosofía de la ciencia natural, Madrid: Alianza.

IMBERNON, F. (1994). La formación y el desarrollo profesional del profesorado. $\mathrm{Ha}$ cia una nueva cultura profesional. BarceIona: Graó

Maturana, H. y Varela, F. (1984). El árbol del conocimiento. Santiago de Chile: Editorial Universitaria.

Ricoeur, P., (1988). Hermenéutica y acción Buenos Aires: Docencia.

SCHÖN, D. (1998). El profesional reflexivo. Barcelona: Paidós.

Tenti Fanfani, E. (1995). "Una carrera con obstáculos: la profesionalización docente". En: Documento de Trabajo del Simposio In ternacional Formación Docente, Modernización Educativa y Globalización, México. 